\title{
Sufficient and Necessary Conditions for the Permanence of a Discrete Model with Beddington-DeAngelis Functional Response
}

\author{
Yong-Hong Fan and Lin-Lin Wang \\ School of Mathematics and Statistics Science, Ludong University, Yantai, Shandong 264025, China \\ Correspondence should be addressed to Yong-Hong Fan; fanyh_1993@sina.com
}

Received 18 December 2013; Revised 20 March 2014; Accepted 24 March 2014; Published 24 April 2014

Academic Editor: Chun-Lei Tang

Copyright ( $\odot 2014$ Y.-H. Fan and L.-L. Wang. This is an open access article distributed under the Creative Commons Attribution License, which permits unrestricted use, distribution, and reproduction in any medium, provided the original work is properly cited.

\begin{abstract}
We give a sufficient and necessary condition for the permanence of a discrete model with Beddington-DeAngelis functional response with the form $x(n+1)=x(n) \exp \{a(n)-b(n) x(n)-c(n) y(n) /(\alpha(n)+\beta(n) x(n)+\gamma(n) y(n))\}, y(n+1)=y(n) \exp \{-d(n)+$ $f(n) x(n) /(\alpha(n)+\beta(n) x(n)+\gamma(n) y(n))\}$, where $a(n), b(n), c(n), d(n), f(n), \alpha(n), \beta(n)$, and $\gamma(n)$ are periodic sequences with the common period $\omega ; b(n)$ is nonnegative; $c(n), d(n), f(n), \alpha(n), \beta(n)$, and $\gamma(n)$ are positive. It is because of the difference between the comparison theorem for discrete system and its corresponding continuous system that an additional condition should be considered. In addition, through some analysis on the limit case of this system, we find that the sequence $\alpha(n)$ has great influence on the permanence.
\end{abstract}

\section{Introduction}

Many mathematical models have been established to describe the relationships between the species and the outer environment or among the different species in biomathematics. The dynamics of the growth of a population can be described if the functional behavior of the rate of growth is known. Of course, it is this functional behavior which is usually measured in the laboratory or in the field. Among the relationships between the species living in the same outer environment, the predator-prey theory plays an important and fundamental role. The dynamic relationship between predators and their prey has long been and will continue to be one of the dominant themes in both ecology and mathematical ecology due to its universal existence and importance. These problems may appear to be simple mathematically at first sight; they are, in fact, very challenging and complicated. Though most predator-prey theories are based on continuous models governed by differential equations, the discrete time models are more appropriate than the continuous ones when the size of the population is rarely small or the population has nonoverlapping generations. On the other hand, the concept of permanence has played an important role in mathematical ecology. Biologically, when a system of interacting species is persistent in a suitable sense, it means that all the species survive in the long term. For investigations on permanence of discrete predator-prey models, one can refer to [1-3] and references cited therein.

In 2006, Cui and Takeuchi studied the permanence, extinction, and periodic solutions for a predator-prey model with Beddington-DeAngelis functional response (see [4]); they gave a sufficient and necessary condition to guarantee the predator and prey to be permanent. As we all know, the continuous dynamic system and its corresponding discrete dynamic system, in some extent, have some similar properties, but also they have many differences. In this paper, we want to study the permanence for the following discrete predator-prey model with Beddington-DeAngelis functional response:

$$
\begin{aligned}
& x(n+1) \\
& =x(n) \exp \{a(n)-b(n) x(n) \\
& \left.\quad-\frac{c(n) y(n)}{\alpha(n)+\beta(n) x(n)+\gamma(n) y(n)}\right\},
\end{aligned}
$$




$$
\begin{aligned}
y(n+1) & \\
=y(n) \exp \{-d(n) & \\
& \left.+\frac{f(n) x(n)}{\alpha(n)+\beta(n) x(n)+\gamma(n) y(n)}\right\},
\end{aligned}
$$

where $a(n), b(n), c(n), d(n), f(n), \alpha(n), \beta(n)$, and $\gamma(n)$ are periodic sequences with the common period $\omega ; b(n)$ is nonnegative; $c(n), d(n), f(n), \alpha(n), \beta(n)$, and $\gamma(n)$ are positive. The reason and significance for the analysis on the properties of these biological models could be found in $[5,6]$. The system (1) can be seen as the discrete form of the continuous situation which has been investigated in [4]. And the discretization method could be found in [7].

As usual, we define the average value of periodic sequences with period $\omega$ as

$$
A_{\omega}(f(n))=\frac{1}{\omega} \sum_{n=0}^{\omega-1} f(n),
$$

and we denote

$$
f^{M}=\max _{n \in I_{\omega}} f(n), \quad f^{L}=\min _{n \in I_{\omega}} f(n),
$$

where $I_{\omega}=\{0,1,2, \ldots \omega-1\}$.

In order to describe our main results, we need some lemmas below.

Lemma 1. If $b(n) \geq 0$ for all $n \in R$ and $A_{\omega}(b(n))>0$, then $x(n+1)=x(n) \exp \{a(n)-b(n) x(n)\}$ has at least one nonnegative $\omega$-periodic solution $x^{*}(n)$. Moreover, if $A_{\omega}(a(n))>0$, then $x^{*}(n)>0$ for all $n \in R$ and if $A_{\omega}(a(n)) \leq 0$, then $x^{*}(n) \equiv 0$. Moreover, if $A_{\omega}(a(n)) \leq 0$, then the zero solution is globally asymptotically stable for any positive initial condition.

Proof. The existence conclusion could be found in [8]. Now we only prove the globally asymptotical stability. That is, we consider the case $A_{\omega}(a(n)) \leq 0$. Notice that

$$
\ln \frac{x(n+1)}{x(n)}=a(n)-b(n) x(n),
$$

which implies that

$$
\begin{aligned}
\sum_{i=0}^{n} \ln \frac{x(i+1)}{x(i)} & =\sum_{i=0}^{n} a(i)-\sum_{i=0}^{n} b(i) x(i) \\
& <\sum_{i=0}^{n} a(i), \quad \text { for } n \text { sufficiently large. }
\end{aligned}
$$

Therefore

$$
\begin{aligned}
x(n+1) & \leq x(0) \exp \left\{\sum_{i=0}^{n} a(i)\right\} \\
& \leq x(0) \exp \left\{\left[\frac{n}{\omega}\right] \sum_{i=0}^{\omega-1} a(i)+\sum_{i=0}^{\omega-1}|a(i)|\right\},
\end{aligned}
$$

where $[n / \omega]$ represents the integer part of $n / \omega$; thus if $A_{\omega}(a(n))<0$, then $\lim _{n \rightarrow \infty} x(n)=0$. If $A_{\omega}(a(n))=0$, by (4), we have

$$
\begin{aligned}
x(n(\omega-1)+i)= & x((n-1)(\omega-1)+i) \\
& \times \exp \left\{\sum_{j=(n-1)(w-1)+i}^{n(\omega-1)+i} a(j)\right. \\
& \left.-\sum_{j=(n-1)(w-1)+i}^{n(\omega-1)+i} b(j) x(j)\right\} \\
\leq & x((n-1)(\omega-1)+i), \quad i \in I_{\omega},
\end{aligned}
$$

which implies that all the subsequences $\{x(n(\omega-1)+i)\}$ of $\{x(n)\}$ are monotonically decreasing. Notice that they all have a lower bounded zero; thus, there exists some nonnegative constant $A_{i}$ such that $\lim _{n \rightarrow \infty} x(n(\omega-1)+i)=A_{i}\left(i \in I_{\omega}\right)$. We claim that all $A_{i}=0$. If all $A_{i} \neq 0$, then $A=\min _{1 \leq i \leq \omega-1} A_{i}>$ 0 ; for $n$ sufficiently large, we have $\sum_{j=(n-1)(w-1)+i}^{n(\omega-1)+i} b(j) x(j) \geq$ $(A / 2) \sum_{j=(n-1)(w-1)+i}^{n(\omega-1)+i} b(j)=(A \omega / 2) A_{\omega}(b(n))>0$. While $(7)$ implies that

$$
\lim _{n \rightarrow \infty} \sum_{j=(n-1)(w-1)+i}^{n(\omega-1)+i} b(j) x(j)=0,
$$

this contradiction shows that there exists at least one $i \in I_{\omega}$ such that

$$
A_{i}=0 \text {, }
$$

and then (4) implies that all $A_{i}=0$. The proof is complete.

Remark 2. This lemma is different from the continuous one; here the condition $A_{\omega}(a(n))>0$ can not support the globally asymptotical stability of $x^{*}(n)$ (from the work of May [9] and Zhang and Zhou [10]). In addition, we can find that the continuous form of this lemma plays an important role in the proof of the permanence in [4].

Lemma 3. Assume that $a(n)$ and $b(n)$ are all $\omega$-periodic sequences and $b(n)$ is positive; if $A_{\omega}(a(n))>0$ and the following inequality

$$
\left[\frac{b(n+1)}{b(n)} \exp \{a(n)-1\}\right]^{M} \leq 2
$$

holds, then any solution $x(n)$ for the periodic equation $x(n+$ $1)=x(n) \exp \{a(n)-b(n) x(n)\}$ with positive initial condition has the property

$$
\lim _{n \rightarrow \infty}\left(x(n)-x^{*}(n)\right)=0,
$$

where $x^{*}(n)$ is defined as that in Lemma 1.

Proof. By Lemma 1, we know that $x^{*}(n)$ exists, and it is positive; for any positive solution $x(n)$ of the equation

$$
x(n+1)=x(n) \exp \{a(n)-b(n) x(n)\},
$$


denote

$$
B(n)=\ln \frac{x(n)}{x^{*}(n)},
$$

and then $B(n)$ satisfies

$$
B(n+1)-B(n)=b(n) x^{*}(n)[1-\exp \{B(n)\}] .
$$

Define $V(n)=B^{2}(n)$, and then

$$
\begin{aligned}
\Delta V(n)= & V(n+1)-V(n) \\
= & {[B(n+1)-B(n)][B(n+1)+B(n)] } \\
= & b(n) x^{*}(n)[1-\exp \{B(n)\}] \\
& \times\left[2 B(n)+b(n) x^{*}(n)[1-\exp \{B(n)\}]\right] \\
= & -B^{2}(n) b(n) x^{*}(n) \exp \{\theta B(n)\} \\
& \times\left[2-b(n) x^{*}(n) \exp \{\theta B(n)\}\right] .
\end{aligned}
$$

Notice that

$$
\begin{aligned}
b(n+1) & x(n+1) \\
& =\frac{b(n+1)}{b(n)} b(n) x(n) \exp \{a(n)-b(n) x(n)\} \\
& \leq \frac{b(n+1)}{b(n)} \exp \{a(n)-1\}, \quad \text { for any } n \geq 1,
\end{aligned}
$$

and here we use the inequality $x \exp \{a-x\} \leq \exp \{a-1\}$; thus for any positive solution $x(n)$ of (12), we have

$$
b(n) x(n) \leq\left[\frac{b(n+1)}{b(n)} \exp \{a(n)-1\}\right]^{M},
$$

and by (10), we know that

$$
b(n) x(n) \leq 2 .
$$

In particular, $b(n) x^{*}(n) \leq 2$. Then equality (15) implies that $\Delta V(n) \leq 0$; that is, $B^{2}(n)$ is nonincreasing; thus it converges, by $(14), \lim _{n \rightarrow \infty} B(n)=0$. The proof is complete.

Remark 4. In [11], Professor Zhou considered the existence and stability of the periodic solution of the equation $x(n+1)=$ $x(n) \exp \{r(n)(1-(x(n) / K(n)))\}$. Here $r(n)$ and $K(n)$ are all positive $\omega$-periodic sequences; under the condition

$$
\frac{K^{M}}{K^{L}} \exp \left(r^{M}-1\right) \leq 2,
$$

the conclusion of Lemma 3 is satisfied. By Lemma 3, the condition (19) can be replaced by

$$
\left[\frac{K(n) r(n+1)}{K(n+1) r(n)} \exp (r(n)-1)\right]^{M} \leq 2 .
$$

Notice that if $K(n) \equiv 1$, then the condition (19) can be simplified as

$$
[r(n)]^{M} \leq \ln 2+1
$$

In fact, in this case, by the work of Zhang and Zhou [10], the condition (21) could be generalized to

$$
[r(n)]^{M} \leq 2
$$

It is worthy to say that, when $[a(n)]^{L}>2$, the conclusion of Lemma 3 is false. This is quite different from the corresponding continuous case. In particular, if $a(n) / b(n) \equiv$ constant, then (10) could be replaced by (22).

For the permanence of (1), we have the following.

Theorem 5. Assume that $a(n)$ and $b(n)$ are all $\omega$-periodic sequences and $b(n)$ is positive; if

$$
\left[\frac{b(n+1)}{b(n)} \exp \{a(n)-1\}\right]^{M} \leq 1
$$

and $A_{\omega}(a(n))>0$ hold true, then the system (1) is permanent and has at least one positive $\omega$-periodic solution provided that

$$
A_{\omega}\left(-d(n)+\frac{f(n) x^{*}(n)}{\alpha(n)+\beta(n) x^{*}(n)}\right)>0,
$$

where $x^{*}(n)$ is the unique periodic solution of $x(n+1)=$ $x(n) \exp \{a(n)-b(n) x(n)\}$ given by Lemma 1.

Theorem 6. Suppose that

$$
A_{\omega}(a(n))>0, \quad A_{\omega}(b(n))>0
$$

and (23) hold; if (1) is permanent, then (24) is true.

By Theorems 5 and 6, we can easily obtain the following.

Corollary 7. Assume that $a(n)$ and $b(n)$ are all $\omega$-periodic sequences and $b(n)$ is positive; if $(23)$ and $A_{\omega}(a(n))>0$ hold true, then system (1) is permanent if and only if (24) holds.

\section{Proof of the Main Results}

In this section, we will give the proofs of Theorems 5 and 6 . First we give some lemmas.

Lemma 8. Under the condition (25), there exist two positive constants $M_{x}$ and $M_{y}$ such that

$$
\lim _{n \rightarrow \infty} \sup x(n) \leq M_{x}, \quad \lim _{n \rightarrow \infty} \sup y(n) \leq M_{y}
$$

for any solution $(x(n), y(n))$ of (1) with positive initial conditions.

Proof. Notice that, for any positive initial value, from mathematical induction, we can obtain that $x(n)>0, y(n)>0$. Then we have

$$
x(n+1)<x(n) \exp \{a(n)-b(n) x(n)\} .
$$

Notice that

$$
x^{*}(n+1)=x^{*}(n) \exp \left\{a(n)-b(n) x^{*}(n)\right\} .
$$


If we let

$$
\begin{gathered}
x(n)=\exp \{u(n)\}, \quad x^{*}(n)=\exp \left\{u^{*}(n)\right\}, \\
v(n)=u(n)-u^{*}(n),
\end{gathered}
$$

then by (27) and (28), we have

$$
v(n+1)-v(n)<-b(n) \exp \left\{u^{*}(n)\right\}[\exp \{v(n)\}-1] .
$$

If there exists some positive integer $N$ such that $v(n)<0$ for $n>N$, then $\lim _{n \rightarrow \infty} \sup x(n) \leq\left[x^{*}(n)\right]^{M}$. If there exists some positive integer $N$ such that $v(n)>0$ for $n>$ $N$, then (30) implies that the sequence $\{v(n)\}$ converges to zero, which shows that $\lim _{n \rightarrow \infty} \sup \left(x(n)-x^{*}(n)\right)=0$; thus $\lim _{n \rightarrow \infty} \sup x(n) \leq\left[x^{*}(n)\right]^{M}$ also hold true. If the sequence $\{v(n)\}$ oscillates about zero, let $v\left(n_{l}\right)$ be the first element of the $l$ th positive semicycle of the sequence $\{v(n)\}$; then from (30), we have

$$
\begin{aligned}
& \lim _{n \rightarrow \infty} \sup v(n)=\lim _{l \rightarrow \infty} v\left(n_{l}\right) \\
& v\left(n_{l}\right)-v\left(n_{l}-1\right)<-b\left(n_{l}-1\right) \exp \left\{u^{*}\left(n_{l}-1\right)\right\} \\
& \times\left[\exp \left\{v\left(n_{l}-1\right)\right\}-1\right] \\
&=-b\left(n_{l}-1\right) \exp \left\{u\left(n_{l}-1\right)\right\} \\
&+b\left(n_{l}-1\right) \exp \left\{u^{*}\left(n_{l}-1\right)\right\} \\
& \leq b\left(n_{l}-1\right) \exp \left\{u^{*}\left(n_{l}-1\right)\right\} \\
& \leq {\left[b(n) \exp \left\{u^{*}(n)\right\}\right]^{M}, }
\end{aligned}
$$

and therefore

$$
\lim _{l \rightarrow \infty} v\left(n_{l}\right) \leq\left[b(n) \exp \left\{u^{*}(n)\right\}\right]^{M},
$$

and from the above analysis, we can obtain

$$
\lim _{n \rightarrow \infty} \sup x(n) \leq\left[x^{*}(n)\right]^{M}+\left[b(n) x^{*}(n)\right]^{M}:=M_{x} .
$$

From the second equation of (1), we have

$$
\begin{aligned}
& y(n+1) \\
& \leq y(n) \exp \{-d(n) \\
& \left.+\frac{f(n) x(n)}{\alpha(n)+\beta(n) x(n)+\gamma(n) y(n)}\right\} \\
& \leq y(n) \exp \left\{-d^{L}+\frac{f^{M} M_{x}}{\alpha^{L}+\gamma^{L} y(n)}\right\} \\
& =y(n) \exp \left\{\frac{f^{M} M_{x}-\alpha^{L} d^{L}-\gamma^{L} d^{L} y(n)}{\alpha^{L}+\gamma^{L} y(n)}\right\} \\
& <y(n) \exp \left\{\frac{f^{M} M_{x}-\gamma^{L} d^{L} y(n)}{\alpha^{L}+\gamma^{L} y(n)}\right\} .
\end{aligned}
$$

If $\{y(n)\}$ does not oscillate about $f^{M} M_{x} / \gamma^{L} d^{L}$, then from (34) we have

$$
\lim _{n \rightarrow \infty} \sup y(n) \leq \frac{f^{M} M_{x}}{\gamma^{L} d^{L}} .
$$

Otherwise, if we let $y\left(n_{l}\right)$ be the first element of the $l$ th positive semicycle of the sequence $\{y(n)\}$, then from (34), we know that

$$
\lim _{n \rightarrow \infty} \sup y(n)=\lim _{l \rightarrow \infty} y\left(n_{l}\right) \leq \frac{f^{M} M_{x}}{\gamma^{L} d^{L}} \exp \left\{\frac{f^{M} M_{x}}{\alpha^{L}}\right\},
$$

and by (35) and (36), we have

$$
\lim _{n \rightarrow \infty} \sup y(n) \leq \frac{f^{M} M_{x}}{\gamma^{L} d^{L}} \exp \left\{\frac{f^{M} M_{x}}{\alpha^{L}}\right\}:=M_{y} .
$$

The proof is complete.

Lemma 9. Assume that (25) holds true, then there exists a positive constant $L_{x}$ such that

$$
\lim _{n \rightarrow \infty} \sup x(n) \geq L_{x}
$$

for any solution $(x(n), y(n))$ of (1) with positive initial conditions.

Proof. We prove it by contradiction. If (38) is false, then for every $m>0$, there exists a solution $\left(x\left(n, z_{m}\right), y\left(n, z_{m}\right)\right)$ with initial condition $\left(x\left(0, z_{m}\right), y\left(0, z_{m}\right)\right)$ such that

$$
\lim _{n \rightarrow \infty} \sup x\left(n, z_{m}\right)<\frac{1}{m} \text {. }
$$

Choose sufficiently small positive constants $\varepsilon_{x}<1$ and $\varepsilon_{y}<1$ such that

$$
\begin{aligned}
& A_{\omega}\left(-d(t)+\frac{f(n) \varepsilon_{x}}{\alpha(n)+\beta(n) \varepsilon_{x}}\right)<0, \\
& A_{\omega}\left(a(n)-\frac{c(n) \varepsilon_{y}}{\alpha(n)+\gamma(n) \varepsilon_{y}}\right)>0 .
\end{aligned}
$$

From (39), for any given $\varepsilon_{x}<1$, there exists a positive integer $N_{0}$ such that

$$
\lim _{n \rightarrow \infty} \sup x\left(n, z_{m}\right)<\frac{1}{m}<\varepsilon_{x}, \quad m>N_{0}
$$

Equation (42) shows that there exists a sufficiently large $N_{1}^{(m)}$ such that

$$
x\left(n, z_{m}\right)<\frac{1}{m}<\varepsilon_{x}, \quad \text { for } m>N_{0}, n \geq N_{1}^{(m)} .
$$

Then the second equation of (1) now yields

$$
y\left(n+1, z_{m}\right) \leq y\left(n, z_{m}\right) \exp \left\{-d(n)+\frac{f(n) \varepsilon_{x}}{\alpha(n)+\beta(n) \varepsilon_{x}}\right\},
$$


and by (40), we have

$$
\begin{aligned}
& \ln \frac{y\left((k+1) \omega, z_{m}\right)}{y\left(k \omega, z_{m}\right)} \\
& <\omega A_{\omega}\left(-d(n)+\frac{f(n) \varepsilon_{x}}{\alpha(n)+\beta(n) \varepsilon_{x}}\right)<0, \\
& \text { for } k \geq N_{1}^{(m)} .
\end{aligned}
$$

This implies that the subsequence $\left\{y\left(k \omega, z_{m}\right)\right\}$ of $\left\{y\left(n, z_{m}\right)\right\}$ is monotonically decreasing; thus it is convergent; by (45),

$$
\lim _{k \rightarrow \infty} y\left(k \omega, z_{m}\right)=0 .
$$

Notice that $\exp \left\{-d(n)+f(n) \varepsilon_{x} /\left(\alpha(n)+\beta(n) \varepsilon_{x}\right)\right\}$ is bounded; thus by (44), using mathematical induction, we can easily obtain

$$
\lim _{n \rightarrow \infty} y\left(n, z_{m}\right)=0
$$

and thus there exists a sufficiently large $N_{2}^{(m)}>N_{1}^{(m)}$ such that

$$
y\left(n, z_{m}\right)<\varepsilon_{y} \text { for } n>N_{2}^{(m)}
$$

therefore the first equation of (1) yields

$$
\begin{aligned}
& x\left(n+1, z_{m}\right) \\
& \geq x\left(n, z_{m}\right) \exp \left\{a(n)-b(n) x\left(n, z_{m}\right)\right. \\
& \left.\quad-\frac{c(n) \varepsilon_{y}}{\alpha(n)+\gamma(n) \varepsilon_{y}}\right\}, \quad n>N_{2}^{(m)} .
\end{aligned}
$$

By (41), utilizing Lemma 1 , we know that the equation $P(n+$ $1)=P(n) \exp \left\{a(n)-c(n) \varepsilon_{y} /\left(\alpha(n)+\gamma(n) \varepsilon_{y}\right)-b(n) P(n)\right\}$ has at least one positive $\omega$-periodic solution called $P^{*}(n)$. It is obvious that $P^{*}(n)$ is independent of $z_{m}$. Let

$$
\begin{gathered}
P^{*}(n)=\exp \left\{Q^{*}(n)\right\}, \quad x\left(n, z_{m}\right)=\exp \left\{Q\left(n, z_{m}\right)\right\}, \\
H\left(n, z_{m}\right)=Q\left(n, z_{m}\right)-Q^{*}(n),
\end{gathered}
$$

then

$$
\begin{aligned}
H\left(n+1, z_{m}\right) \geq & H\left(n, z_{m}\right)-b(n) \exp \left\{Q^{*}(n)\right\} \\
& \times\left[\exp \left\{H\left(n, z_{m}\right)\right\}-1\right] .
\end{aligned}
$$

If $H\left(n, z_{m}\right)$ does not oscillate about zero, then

$$
\lim _{n \rightarrow \infty} \inf H\left(n, z_{m}\right) \geq 0
$$

which implies that when $n$ is large enough,

$$
x\left(n, z_{m}\right) \geq P^{*}(n) \geq\left[P^{*}(n)\right]^{L}>0 .
$$

If $H\left(n, z_{m}\right)$ oscillates about zero, let $H\left(n_{l}, z_{m}\right)$ be the first element of the $l$ th negative semicycle of the sequence $\left\{H\left(n, z_{m}\right)\right\}$; then from (51), we know that

$$
\lim _{n \rightarrow \infty} \inf H\left(n, z_{m}\right)=\lim _{l \rightarrow \infty} H\left(n_{l}, z_{m}\right) .
$$

Notice that

$$
\begin{aligned}
H\left(n_{l}, z_{m}\right) \geq & H\left(n_{l}-1, z_{m}\right)-b\left(n_{l}-1\right) \\
& \times \exp \left\{Q^{*}\left(n_{l}-1\right)\right\}\left[\exp \left\{H\left(n_{l}-1, z_{m}\right)\right\}-1\right] \\
\geq & -b\left(n_{l}-1\right) \exp \left\{Q\left(n_{l}-1\right)\right\} \\
\geq & -[b(n)]^{M} M_{x},
\end{aligned}
$$

and thus when $n$ is sufficiently large,

$$
x\left(n, z_{m}\right) \geq\left[P^{*}(n)\right]^{L} \exp \left\{-[b(n)]^{M} M_{x}\right\}>0 .
$$

Inequalities (53) and (56) imply that when $n$ is sufficiently large, $x\left(n, z_{m}\right) \geq\left[P^{*}(n)\right]^{L} \exp \left\{-[b(n)]^{M} M_{x}\right\}$. Since $\left[P^{*}(n)\right]^{L} \exp \left\{-[b(n)]^{M} M_{x}\right\}$ is independent of $m$, then

$$
\lim _{m \rightarrow \infty} \inf x\left(n, z_{m}\right) \geq\left[P^{*}(n)\right]^{L} \exp \left\{-\left[b(n) P^{*}(n)\right]^{M}\right\},
$$

and this contradicts (42). The proof is complete.

Lemma 10. Assume that (25), (24), and (23) hold true; then there exists a positive constant $L_{y}$ such that

$$
\lim _{n \rightarrow \infty} \sup y(n) \geq L_{y}
$$

for any solution $(x(n), y(n))$ of (1) with positive initial values.

Proof. If (58) is not true, then, for any $m>0$, there exists a positive initial value $(x(0, m), y(0, m))$ which may be dependent on $m$ such that

$$
\lim _{n \rightarrow \infty} \sup y(n, m) \leq \frac{1}{m}
$$

where $(x(n, m), y(n, m))$ is the solution of (1) with positive initial values $(x(0, m), y(0, m))$.

By (25) and (24), we can choose the constant $\varepsilon_{y}$ and $\varepsilon$ sufficiently small such that

$$
A_{\omega}\left(-d(n)+\frac{f(n)\left(x^{*}(n)-\varepsilon\right)}{\alpha(n)+\beta(n)\left(x^{*}(n)-\varepsilon\right)+\gamma(n) \varepsilon_{y}}\right)>0 \text {, }
$$

$$
A_{\omega}\left(a(n)-\frac{c(n) \varepsilon_{y}}{\alpha(n)+\gamma(n) \varepsilon_{y}}\right)>0 .
$$


From (59), we can choose $m$ sufficiently large such that $1 / m<$ $\varepsilon_{y}$; then the first equation of (1) implies that

$$
\begin{aligned}
x(n+1, m) & \\
\geq & x(n, m) \\
& \times \exp \left\{a(n)-\frac{c(n) \varepsilon_{y}}{\alpha(n)+\gamma(n) \varepsilon_{y}}-b(n) x(n, m)\right\} .
\end{aligned}
$$

By (61) and Lemmas 1 and 3, the following equation

$$
z(n+1)=z(n) \exp \left\{a(n)-\frac{c(n) \varepsilon_{y}}{\alpha(n)+\gamma(n) \varepsilon_{y}}-b(n) z(n)\right\}
$$

has a unique positive $\omega$-periodic solution $z^{*}(n)$ for any sufficiently small positive number $\varepsilon_{y}$ and

$$
\lim _{\varepsilon_{y} \rightarrow 0}\left(z^{*}(n)-x^{*}(n)\right)=0 .
$$

We claim that, for any $\varepsilon>0$, there exists a sufficiently large $N$ such that

$$
x(n, m) \geq x^{*}(n)-\varepsilon, \quad \text { for } n>N .
$$

In fact, by (62) and (63), if we set

$$
x(n, m)=\exp \{U(n, m)\}, \quad z(n)=\exp \{V(n)\},
$$

where the sequence $\{z(n)\}$ is the solution of (63) with initial condition $z(0)=x(0, m)$, then

$$
\begin{aligned}
U(n+1, m) \geq & U(n, m)+a(n) \\
& -\frac{c(n) \varepsilon_{y}}{\alpha(n)+\gamma(n) \varepsilon_{y}}-b(n) \exp \{U(n, m)\}, \\
V(n+1)= & V(n)+a(n) \\
& -\frac{c(n) \varepsilon_{y}}{\alpha(n)+\gamma(n) \varepsilon_{y}}-b(n) \exp \{V(n)\} .
\end{aligned}
$$

Thus

$$
\begin{aligned}
U(n+ & 1, m)-V(n+1) \\
\geq & U(n, m)-V(n) \\
& \quad-b(n) \exp \{V(n)\}\{\exp \{U(n, m)-V(n)\}-1\} .
\end{aligned}
$$

Denote

$$
W(n, m)=U(n, m)-V(n),
$$

and then (68) implies that

$$
\begin{aligned}
W(n+1, m) \geq & W(n, m) \\
& -b(n) \exp \{V(n)\}[\exp \{W(n, m)\}-1] .
\end{aligned}
$$

Notice that

$$
x(n+1, m) \leq x(n, m) \exp \{a(n)-b(n) x(n, m)\},
$$

and by (23), we have

$$
\begin{aligned}
b(n+1) & x(n+1, m) \\
\leq & \frac{b(n+1)}{b(n)} b(n) x(n, m) \exp \{a(n)-b(n) x(n, m)\} \\
& \leq\left[\frac{b(n+1)}{b(n)} \exp \{a(n)-1\}\right]^{M} \leq 1, \quad \text { for any } n \in N .
\end{aligned}
$$

Define a function

$$
f(x)=x-h(\exp \{x\}-1), \quad h \exp \{x\} \leq 1 .
$$

Then

$$
f^{\prime}(x)=1-h \exp \{x\} \geq 0 .
$$

From (70), we can obtain $W(1, m) \geq W(0, m)=0$. By $(73)$, (74), and (72), we know that

$$
W(n, m) \geq 0,
$$

which implies that

$$
x(n, m) \geq z(n) .
$$

Under condition (23), by Lemma 3, we have

$$
\lim _{n \rightarrow \infty}\left(z(n)-z^{*}(n)\right)=0
$$

and then (64), (77), and (76) imply that (65) holds. Now the second equation of (1) yields

$$
\begin{aligned}
y(n+1, m) \\
\geq y(n, m) \\
\quad \times \exp \left\{-d(n)+\frac{f(n)\left(x^{*}(n)-\varepsilon\right)}{\alpha(n)+\beta(n)(x(n)-\varepsilon)+\gamma(n) \varepsilon_{y}}\right\},
\end{aligned}
$$

and thus (60) shows that

$$
\lim _{n \rightarrow \infty} \sup y(n, m)=+\infty
$$

This contradicts (59). The proof is complete.

Lemma 11. Assume that (25) holds true; then there is a positive constant $L_{x}^{\prime}$ such that

$$
\lim _{n \rightarrow \infty} \inf x(n) \geq L_{x}^{\prime}
$$

for any solution $(x(n), y(n))$ of (1) with positive initial conditions. 
Proof. If (80) is false, then there exists an initial value $(x(0), y(0)) \triangleq t_{m}$ such that

$$
\lim _{n \rightarrow \infty} \inf x\left(n, t_{m}\right) \leq \frac{1}{m}
$$

for any $m>0$, where $\left(x\left(n, t_{m}\right), y\left(n, t_{m}\right)\right)$ represents the solution of (1) with initial value $t_{m}$. Thus, there is a subsequence $\left\{n_{l}\right\}$ of $\{n\}$ such that

$$
x\left(n_{l}, t_{m}\right) \leq \frac{1}{m} .
$$

On the other hand, by Lemma 9, there exists a constant $L_{x} / 2$ (which is independent of the initial value $t_{m}$ ) such that

$$
x\left(m_{k}, t_{m}\right) \geq \frac{L_{x}}{2} .
$$

Notice that, for any $n_{l}$, there exists a $m_{k}$ which satisfies $n_{l} \leq$ $m_{k},(82)$, and (83). Choose $m$ sufficiently large such that

$$
\frac{1}{m} \exp \left\{\left(m_{k}-n_{l}\right)[a(n)]^{M}\right\}<\frac{L_{x}}{2}
$$

Obviously, such $m$ exists. Fixing it, and by the first equation of (1), we know

$$
\begin{aligned}
x\left(n_{l}\right. & \left.+1, t_{m}\right) \\
& \leq x\left(n_{l}, t_{m}\right) \exp \left\{[a(n)]^{M}\right\} \leq \frac{1}{m} \exp \left\{[a(n)]^{M}\right\},
\end{aligned}
$$

and by mathematical induction, we can easily obtain

$$
x\left(m_{k}, t_{m}\right) \leq \frac{1}{m} \exp \left\{\left(m_{k}-n_{l}\right)[a(n)]^{M}\right\}<\frac{L_{x}}{2},
$$

and this contradicts (83). The proof is complete.

Lemma 12. Assume that (25), (24), and (23) hold true, then there exists a positive constant $L_{y}^{\prime}$ such that

$$
\lim _{n \rightarrow \infty} \inf y(n) \geq L_{y}^{\prime}
$$

for any solution $(x(n), y(n))$ of (1) with positive initial values.

Proof. If (87) is false, then for any $m>0$, there exist an initial value $(x(0), y(0)) \triangleq s_{m}$ and a positive integer sequence $\left\{n_{l}\right\}$ such that

$$
y\left(n_{l}, s_{m}\right) \leq \frac{1}{m}
$$

where $\left(x\left(n, s_{m}\right), y\left(n, s_{m}\right)\right)$ represents the solution of (1) with initial value $s_{m}$.
From the proof of Lemma 10, we can find that, for any $\varepsilon>0$, when $n$ is sufficiently large, $x\left(n, s_{m}\right) \leq x^{*}(n)+\varepsilon$,

$$
\begin{aligned}
y\left(n_{l}+\right. & \left.1, s_{m}\right) \\
\leq & y\left(n_{l}, s_{m}\right) \\
& \times \exp \left\{-d(n)+\frac{f(n)\left[x^{*}(n)+\varepsilon\right]}{\alpha(n)+\beta(n)\left[x^{*}(n)+\varepsilon\right]}\right\} \\
\leq & y\left(n_{l}, s_{m}\right) \\
& \times \exp \left\{\left[-d(n)+\frac{f(n)\left[x^{*}(n)+\varepsilon\right]}{\alpha(n)+\beta(n)\left[x^{*}(n)+\varepsilon\right]}\right]^{M}\right\} ;
\end{aligned}
$$

here $n_{l}$ is sufficiently large. On the other hand, by Lemma 10 , there also exist a constant $L_{y} / 2$ (which is independent to $m$ ) and a subsequence $\left\{m_{k}\right\}$ of $\{n\}$ such that

$$
y\left(m_{k}, s_{m}\right) \geq \frac{L_{y}}{2} .
$$

The rest of the proof is similar to that of Lemma 11; we omit it here.

Proof of Theorem 5. By Lemmas 8, 11, and 12, we can easily obtain it.

Proof of Theorem 6. Assume that (1) is permanent; then there exist two constants $L$ and $M$ such that

$$
L<x(n)<M, \quad L<y(n)<M
$$

for simplicity, the inequality holds true only for sufficiently large $n$; we omit the explanation of the domain for $n$ in what follows. Choose $L$ sufficiently small such that

$$
\begin{gathered}
A_{\omega}\left(a(n)-\frac{c(n) L}{\alpha(n)+\beta(n) M+\gamma(n) L}\right)>0 \\
{\left[\frac{b(n+1)}{b(n)} \exp \left\{a(n)-\frac{c(n) L}{\alpha(n)+\beta(n) M+\gamma(n) L}-1\right\}\right]^{M}}
\end{gathered}
$$$$
\leq 1
$$

Consider the following equation:

$$
\begin{aligned}
& u(n+1) \\
& =u(n) \exp \left\{a(n)-\frac{c(n) L}{\alpha(n)+\beta(n) M+\gamma(n) L}\right. \\
& \quad-b(n) u(n)\} ;
\end{aligned}
$$

by (93) and Lemma 3, (94) has a unique positive solution $u^{*}(n)$ which is globally asymptotically stable. Obviously,

$$
\lim _{L \rightarrow 0^{+}}\left(u^{*}(n)-x^{*}(n)\right)=0 .
$$


In addition, $u^{*}(n) \leq x^{*}(n)$. Notice that

$$
\begin{aligned}
& x(n+1) \\
& \leq x(n) \exp \{a(n)-b(n) x(n) \\
& \left.\quad-\frac{c(n) L}{\alpha(n)+\beta(n) M+\gamma(n) L}\right\} .
\end{aligned}
$$

Thus

$$
\begin{gathered}
x(n) \leq u^{*}(n), \\
y(n+1) \leq y(n) \exp \left\{-d(n)+\frac{f(n) x^{*}(n)}{\alpha(n)+\beta(n) x^{*}(n)}\right\} .
\end{gathered}
$$

If

$$
A_{\omega}\left(-d(n)+\frac{f(n) x^{*}(n)}{\alpha(n)+\beta(n) x^{*}(n)}\right)<0,
$$

then (98) implies that

$$
\lim _{n \rightarrow \infty} y(n)=0
$$

this is a contradiction.

If

$$
A_{\omega}\left(-d(n)+\frac{f(n) x^{*}(n)}{\alpha(n)+\beta(n) x^{*}(n)}\right)=0,
$$

then the second equation of (1) implies that

$$
\begin{aligned}
& y(n+1) \\
& \quad \leq y(n) \exp \left\{-d(n)+\frac{f(n) x^{*}(n)}{\alpha(n)+\beta(n) x^{*}(n)+\gamma(t) L}\right\},
\end{aligned}
$$

and by (101),

$$
A_{\omega}\left(-d(n)+\frac{f(n) x^{*}(n)}{\alpha(n)+\beta(n) x^{*}(n)+\gamma(t) L}\right)<0 ;
$$

then (102) also implies (100), which also contradicts the permanence. The proof is complete.

\section{Examples and Discussion}

In this section, we give some examples to illustrate our main results and also give some discussions.

Example 1. Let $b(n)=1, a(3 n+1)=0.5, a(3 n+$ $2)=0.2, a(3 n)=0.8$. Then condition (10) holds true; by Lemma 3, we know that the periodic equation $x(n+1)=$ $x(n) \exp \{a(n)-b(n) x(n)\}$ has only one positive 3-periodic solution. By numerical computation, we know $x^{*}(3 n+1)=$ $0.5812, x^{*}(3 n+2)=0.5359, x^{*}(3 n)=0.3830$. See Figure 1 for more assistance.

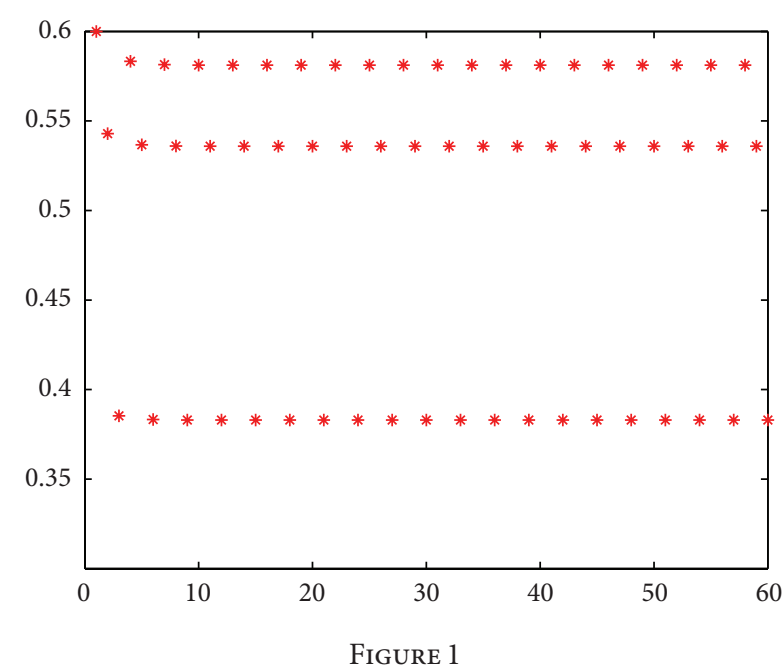

Example 2. Let $c(n)=\gamma(n)=1, a(n)$ and $b(n)$ are defined as in Example 1, $d(3 n+1)=0.2, d(3 n+2)=0.1, d(3 n+3)=$ $0.3, f(3 n+1)=2, f(3 n+2)=1, f(3 n+3)=3, \alpha(3 n+1)=$ $0.1, \alpha(3 n+1)=0.3, \alpha(3 n+1)=0.4, \beta(3 n+1)=0.1, \beta(3 n+1)=$ 0.2 , and $\beta(3 n+1)=0.3$.

By the above definition, we can easily obtain

$$
A_{\omega}\left(-d(n)+\frac{f(n) x^{*}(n)}{\alpha(n)+\beta(n) x^{*}(n)}\right)=3.4330>0,
$$

and by Theorem 5 , we know that system (1) is permanent and has at least one positive 3-periodic solution. In fact, from the numerical results, we can get one of the 3-periodic solutions: $x^{*}(3 n+1)=0.0265, y^{*}(3 n+1)=0.0806, x^{*}(3 n+2)=$ $0.0289, y^{*}(3 n+2)=0.0786, x^{*}(3 n)=0.0364, y^{*}(3 n)=$ 0.0727 . See Figure 2 .

Now let us go back to conditions (10) and (23). Obviously, condition (23) can be included by (10), and condition (10) assures the globally asymptotical stability of the positive periodic solution $x^{*}(n)$ of (12). But under condition (10), for the solution $x(n)$ of the inequality $x(n+1) \leq x(n) \exp \{a(n)-$ $b(n) x(n)\}$, we can not obtain $x(n) \leq x^{*}(n)$ for $n$ sufficiently large; for the solution $x(n)$ of the inequality $x(n+1) \geq$ $x(n) \exp \{a(n)-b(n) x(n)\}$, we can not obtain $x(n) \geq x^{*}(n)$ for $n$ sufficiently large. One crucial reason is that the comparison theorem for discrete system is quite different from its corresponding continuous system. In [8], we gave a qualitative analysis for the permanence of ratio-dependent preypredator model; there we used the comparison theorem of difference equations; unfortunately, an additional condition should be considered, while in [12] we deleted this additional condition (the method we used is not the comparison theorem of difference equations but is the semicycle theory). In the present paper, can condition (23) be deleted? We leave it for further investigation.

In addition, we consider an extreme situation: $\alpha(n) \equiv$ 0 . In this extreme case, we have already obtained the permanence for system (1) (see [12]). Recall that the condition which assures the permanence is $A_{\omega}(f(n)-d(n))>0$ and $A_{\omega}(a(n)-c(n) / \gamma(n))>0$. Let us see the limit 


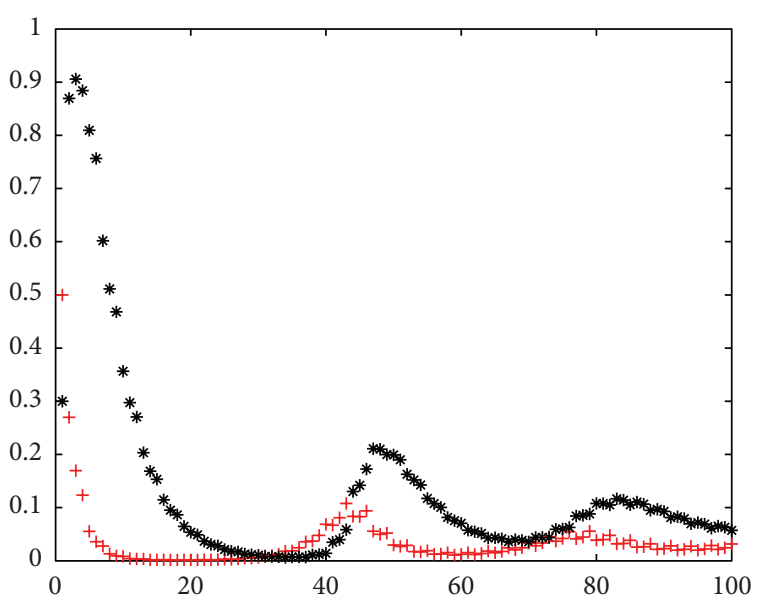

Figure 2

condition under this extreme situation. Now condition (24) was deduced to $A_{\omega}(f(n)-d(n))>0$, while the condition $[(b(n+1) / b(n)) \exp \{a(n)-1\}]^{M} \leq 1$ remains unchanged. Notice that the deduced condition is independent of the sequences $c(n)$ and $\gamma(n)$. Do the two sequences have any influence on the permanence of this extreme situation? The answer is "yes." In [13], we have obtained that when $A_{\omega}(c(n) / \gamma(n))>A_{\omega}(a(n)+d(n))$, the limit system is not persistent. In this point of view, we can see that the sequence $\alpha(n)$ has great influence on the permanence. In fact, from the proof of our main results, we can see that the proof could not be copied to prove the permanence of the limit system (extreme case).

\section{Conflict of Interests}

The authors declare that there is no conflict of interests regarding the publication of this paper.

\section{Acknowledgments}

This work is supported by NSF of China (11201213 and 11371183), NSF of Shandong Province (ZR2010AM022 and ZR2013AM004), and the Outstanding Young and Middleaged Scientists Research Award Fund of Shandong Province (BS2011SF004).

\section{References}

[1] J. Li and J. Yan, "Permanence and extinction for a nonlinear diffusive predator-prey system," Nonlinear Analysis. Theory, Methods \& Applications, vol. 71, no. 1-2, pp. 399-417, 2009.

[2] F. Chen, W. Chen, Y. Wu, and Z. Ma, "Permanence of a stagestructured predator-prey system," Applied Mathematics and Computation, vol. 219, no. 17, pp. 8856-8862, 2013.

[3] K. Wang and Y. Zhu, "Periodic solutions, permanence and global attractivity of a delayed impulsive prey-predator system with mutual interference," Nonlinear Analysis. Real World Applications, vol. 14, no. 2, pp. 1044-1054, 2013.
[4] J. Cui and Y. Takeuchi, "Permanence, extinction and periodic solution of predator-prey system with Beddington-DeAngelis functional response," Journal of Mathematical Analysis and Applications, vol. 317, no. 2, pp. 464-474, 2006.

[5] L. Wang and Y. Fan, "Note on permanence and global stability in delayed ratio-dependent predator-prey models with monotonic functional responses," Journal of Computational and Applied Mathematics, vol. 234, no. 2, pp. 477-487, 2010.

[6] L. Wang and W. Li, "Periodic solutions and permanence for a delayed nonautonomous ratio-dependent predator-prey model with Holling type functional response," Journal of Computational and Applied Mathematics, vol. 162, no. 2, pp. 341-357, 2004.

[7] K. Gopalsamy, Stability and Oscillations in Delay Differential Equations of Population Dynamics, vol. 74, Kluwer Academic Publishers, Dordrecht, The Netherlands, 1992.

[8] Y. Fan and W. Li, "Permanence for a delayed discrete ratiodependent predator-prey system with Holling type functional response," Journal of Mathematical Analysis and Applications, vol. 299, no. 2, pp. 357-374, 2004.

[9] R. May, "Biological populations obeying difference equations: stable points, stable cycles and chaos," Journal of Theoretical Biology, vol. 51, pp. 511-524, 1975.

[10] Q. Zhang and Z. Zhou, "Global attractivity of a nonautonomous discrete logistic model," Hokkaido Mathematical Journal, vol. 29, no. 1, pp. 37-44, 2000.

[11] Z. Zhou and X. Zou, "Stable periodic solutions in a discrete periodic logistic equation," Applied Mathematics Letters, vol. 16, no. 2, pp. 165-171, 2003.

[12] Y. Fan and W. Li, "Harmless delays in a discrete ratio-dependent periodic predator-prey system," Discrete Dynamics in Nature and Society, vol. 2006, Article ID 12176, 21 pages, 2006.

[13] Y. Fan and L. Wang, "Average condition for nonpersistence of a delayed discrete ratio dependent predator-prey system," in Proceedings of the 6th Conference of Biomathematics, vol. 2 of Advances in Biomathematics, pp. 771-774, 2008. 


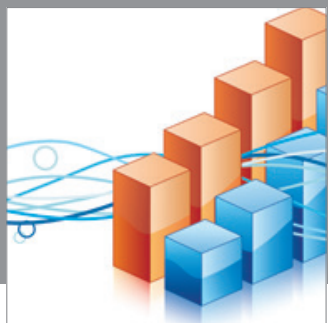

Advances in

Operations Research

mansans

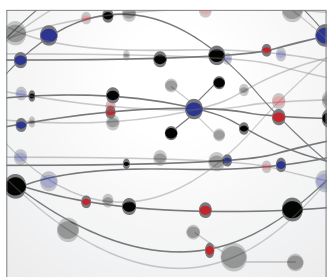

The Scientific World Journal
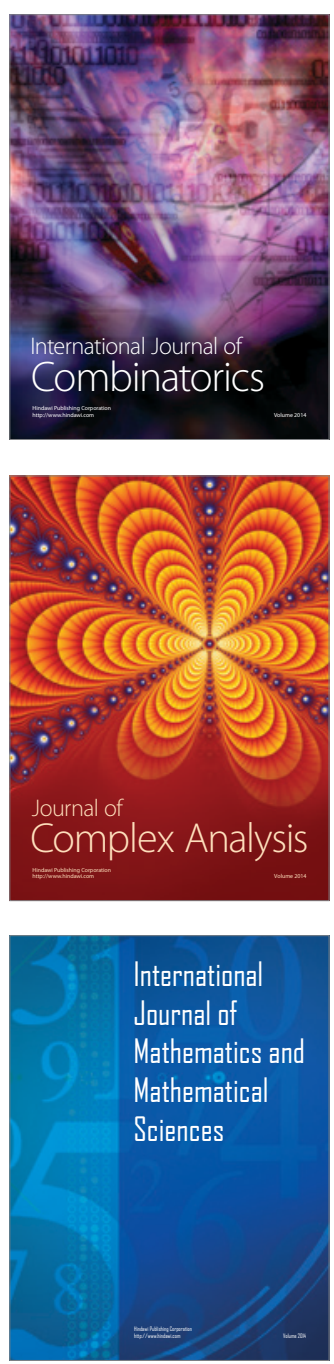
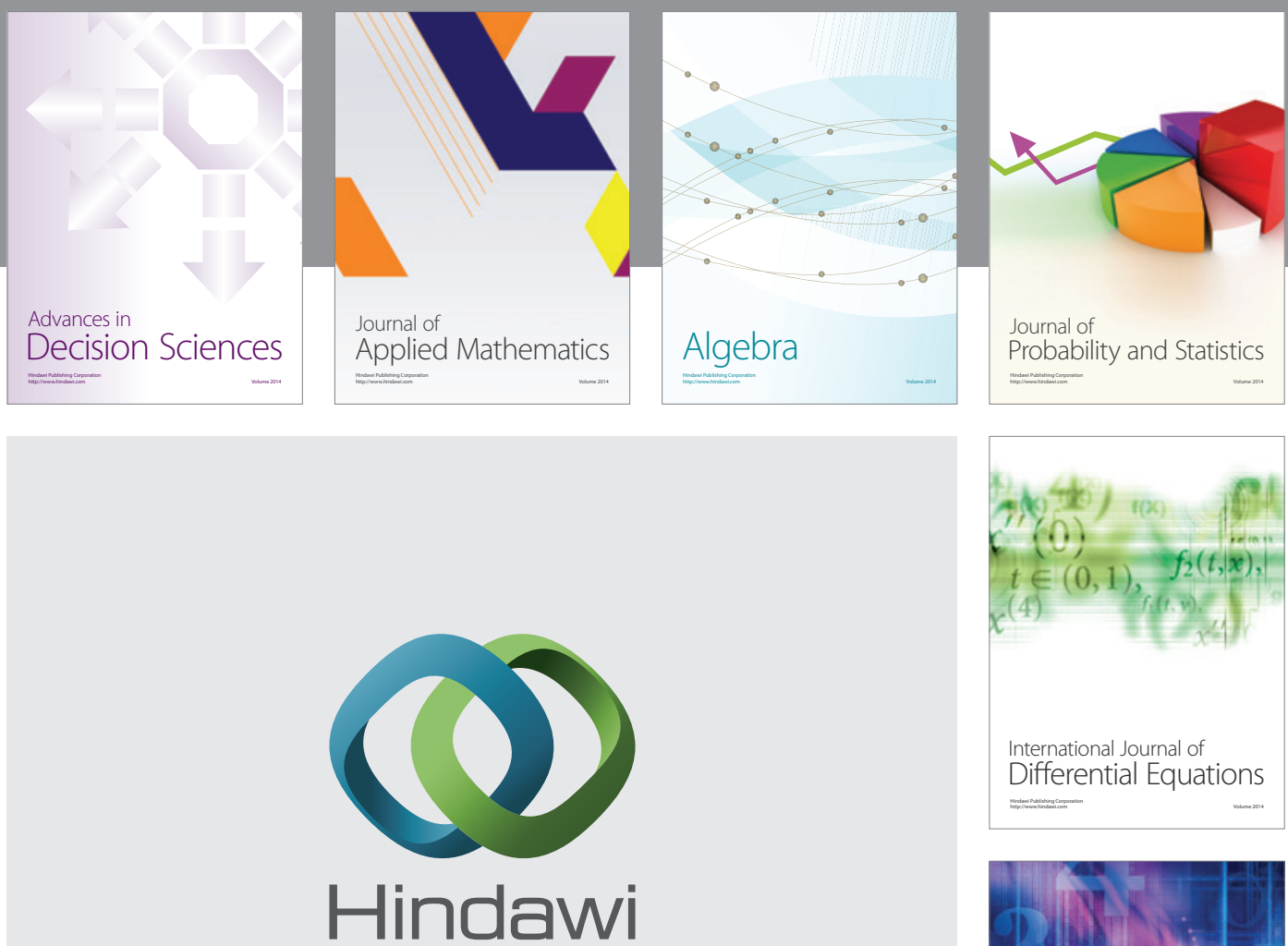

Submit your manuscripts at http://www.hindawi.com
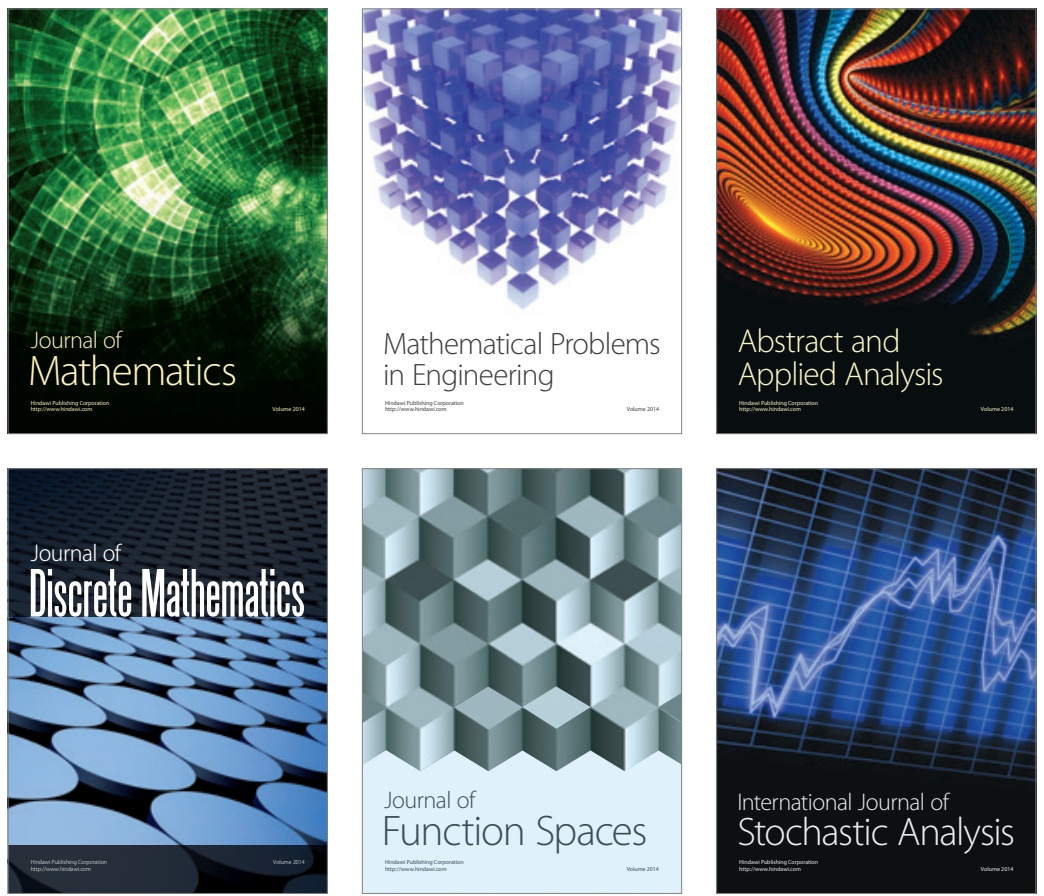

Journal of

Function Spaces

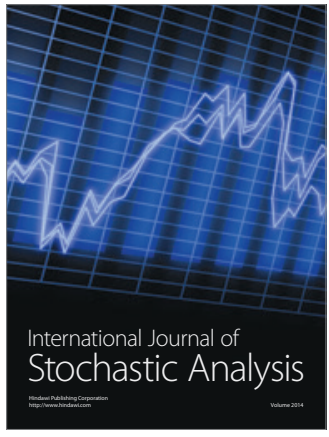

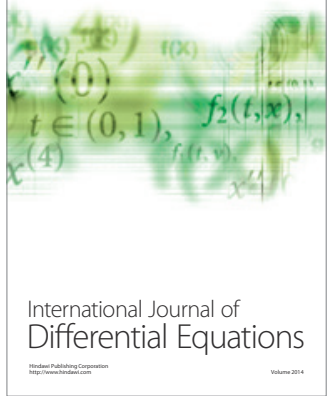
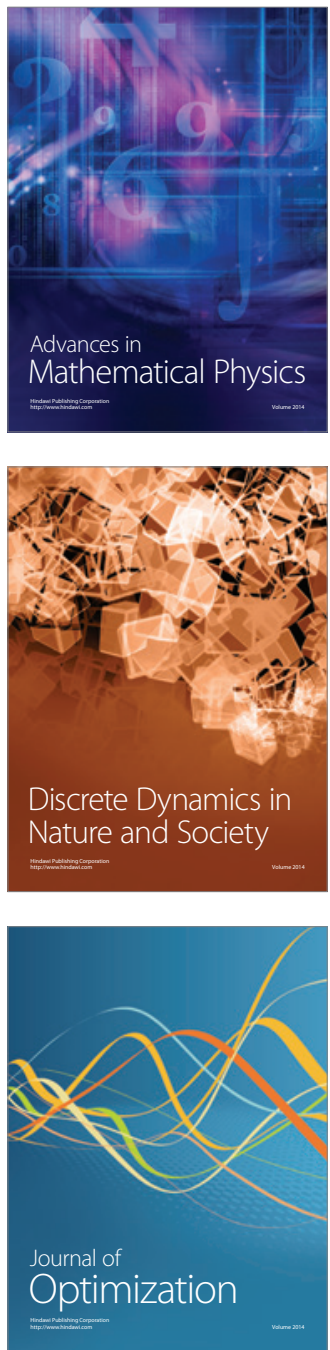